\title{
Bright Electron Beams and Their Applications to Electron Phase Microscopy
}

\author{
Akira TONOMURA
}

Advanced Research Laboratory, Hitachi, Ltd. Hatoyama, Saitama, Japan SORST, Japan Science and Technology Corporation (JST), Tokyo, Japan Frontier Research System, The Institute of Chemical and Physical Research(RIKEN), Saitama, Japan @

Bright beam sources, like lasers and synchrotron radiation, have brought about new ways of investigating microscopic structures. The same is true with electron sources, especially when their phase information is used for microscopy.

We have continued to develop brighter electron beams in order to raise the level of performance of electron microscopy and to enable new applications to it since 1968 [1], when we became convinced through our experience with interference experiments that a bright electron beam was indispensable for obtaining highquality images through electron holography. As it turned out, every time beam brightness was increased, new applications opened up.

In 1978, using a 80-kV field-emission electron beam, we improved beam brightness by two orders of magnitude compared to that of thermal electron beams from pointed filaments, enabling us to directly observe the magnetic lines of force in h/e flux units as an interference micrograph [2]. With our 250-kV microscope, we conclusively confirmed the $\mathrm{AB}$ effect by a series of experiments from 1982 [3] to 1986 [4]. In 1992, we dynamically observed [5] magnetic quantized vortices in superconductors with our 350-kV microscope. In Spring 2000, we completed a 1-MV microscope [6] (Fig. 1) that has a beam brightness higher than that of electron beams from pointed filaments by four orders of magnitude and a lattice resolution of less than $0.5 \AA$.

We have just begun obtaining various new results with this microscope. For example, we have distinguished two kinds of vortices inside Bi-2212 film; one trapped along tilted columnar defects and the other penetrating perpendicularly to the film plane in the form of two different Lorentz images (Fig. 2). We have also obtained information about the experimental conditions where vortices are trapped along the defects and strongly pinned [7]. Furthermore, a special arrangement of vortices, the chain-lattice state in a Bi-2212 thin film, reflecting the layered structure of the material was observed by Lorentz microscopy, and the disappearance of the chain-vortex images was found to occur at temperatures well below the melting temperature [8] (Fig. 3).

With this microscope we will be able to clarify other various unconventional behaviors of vortices in high- $\mathrm{T}_{\mathrm{c}}$ superconductors.

\section{References}

[1] A. Tonomura et. al., Jpn. J. Appl. Phys., 7 (1968) 295.

[2] A. Tonomura et. al., Phys. Rev. Lett. 44 (1980) 1430.

[3] A. Tonomura et al., Phys. Rev. Lett. 48 (1982) 1443.

[4] A. Tonomura et al., Phys. Rev. Lett. 56 (1986) 792.

[5] K. Harada et al., Nature 360 (1992) 51.

[6] T. Kawasaki et al., Appl. Phys. Lett. 76 (2000) 1342.

[7] A. Tonomura et al., Nature 412 (2001) 620.

[8] T. Matsuda et al., Science 294 (2001) 2136. 


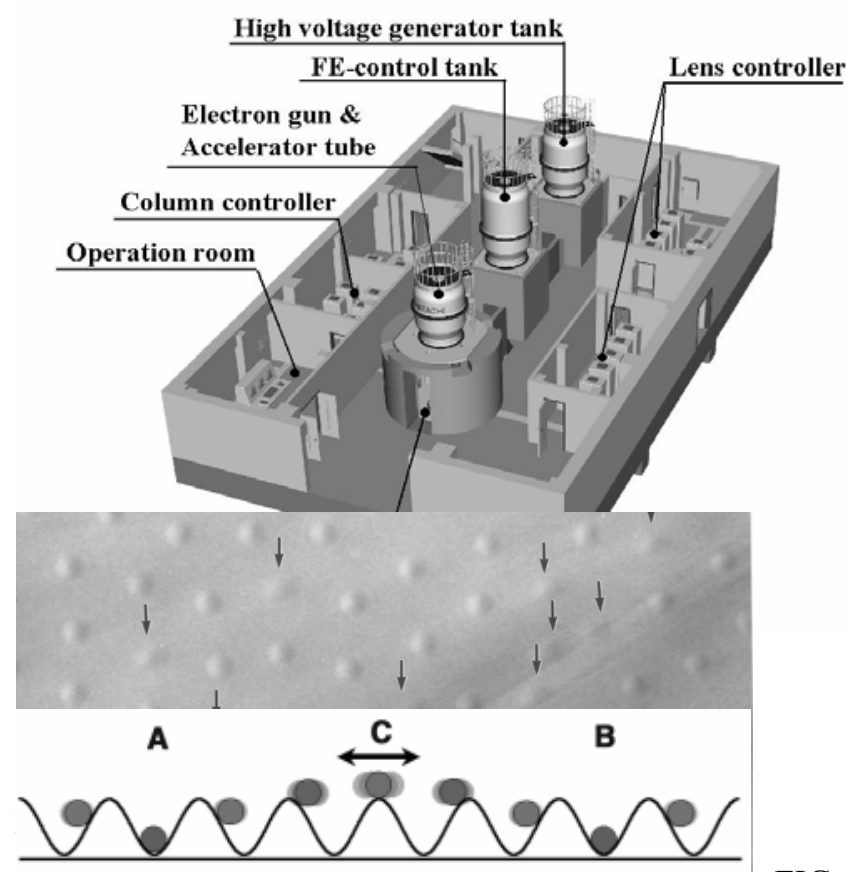

FIG. 1. Schematic of 1-MV field-emission electron microscope [6].

FIG. 3. Disappearance of chain-vortex images in Bi2212 at $70 \mathrm{~K}$ [8]. When a magnetic field was applied at a grazing angle to the layer plane, two domains of chain vortices and lattice vortices were alternately produced. When the temperature was increased, only the images of

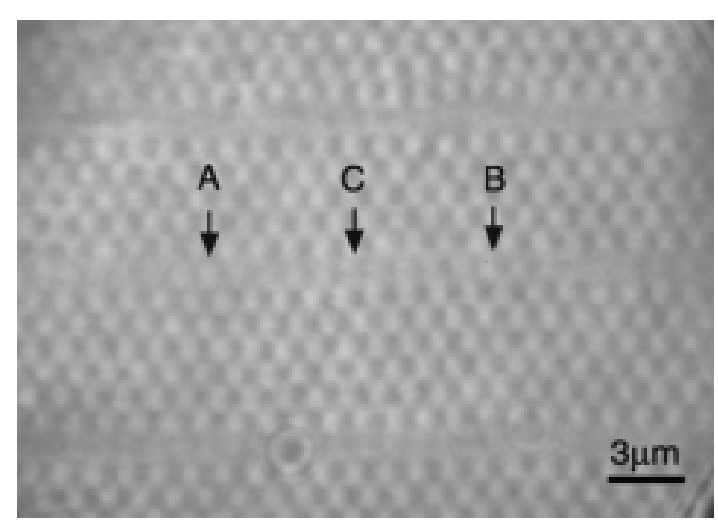
chain vortices begin to disappear. This is attributed to the oscillation of the vortices along the chains. Note that unstable chain vortex $\mathrm{C}$ begins to disappear while stable vortices $\mathrm{A}$ and $\mathrm{B}$ can still be seen. 